Draft Version November 1, 2018

Preprint typeset using $\mathrm{LATEX}_{\mathrm{E}}$ style emulateapj v. 7/8/03

\title{
HOW MERGERS MAY AFFECT THE MASS SCALING RELATION BETWEEN GRAVITATIONALLY BOUND SYSTEMS
}

\author{
Chien Y. Peng ${ }^{1,2}$ \\ (Received 2003 March 13) \\ Draft version November 1, 2018
}

\begin{abstract}
Supermassive black hole $(\mathrm{BH})$ masses $\left(\mathcal{M}_{\mathrm{BH}}\right)$ are strongly correlated with galaxy stellar bulge masses $\left(\mathcal{M}_{\text {bulge }}\right)$ and there are several ideas to explain the origin of this relationship. This study isolates the role of galaxy mergers from considerations of other detailed physics to more clearly show how a linear $\mathrm{BH}-$ galaxy mass relation $\left(\mathcal{M}_{\mathrm{BH}}-\mathcal{M}_{\text {gal }}\right)$ can naturally emerge regardless of how primordial $\mathrm{BHs}$ were seeded inside galaxies, if the galaxy mass function declines with increasing mass. Under this circumstance, the $\mathcal{M}_{\mathrm{BH}^{-}} \mathcal{M}_{\text {gal }}$ relation is a passive attractor that eventually converges to a tight linear relation because of two basic statistical effects: a central limit-like tendency for galaxy mergers which is much stronger for major mergers than for minor mergers, and a convergence towards a linear relation that is due mainly to minor mergers. A curious consequence of this thought experiment is that, if galaxy bulges are formed by major mergers, then merging statistics naturally show that $\mathcal{M}_{\mathrm{BH}}$ would correlate more strongly with bulge dominated galaxies, because of stronger central-seeking tendencies, than with disk dominated galaxies. Even if some other physics is ultimately responsible for causing a linear $\mathcal{M}_{\mathrm{BH}}-\mathcal{M}_{\text {bulge }}$ relationship, this thought experiment shows that, counter to intuition, random merging of galaxies tends to strengthen rather than weaken a pre-existing, linear, correlation. This idea may be generalized to other gravitationally bound systems (dark matter halo, compact nuclear objects) that retain their physical identities after experiencing mergers.
\end{abstract}

Subject headings: galaxies: bulges — galaxies: formation — galaxies: evolution - galaxies: statistics

\section{INTRODUCTION}

In recent years, there have been several surprising discoveries of fundamental scaling relations between supermassive black hole masses $\left(\mathcal{M}_{\mathrm{BH}}\right)$ and large scale galaxy bulge properties: stellar velocity dispersion $\sigma_{*}$, bulge mass $\mathcal{M}_{\text {bulge, }}$ the profile slope of galaxies, and the inner core radius (Gebhardt et al. 2000; Ferrarese \& Merritt 2000; Kormendy \& Richstone 1995; Magorrian et al. 1998; Ho 1999; Kormendy \& Gebhardt 2001; Graham et al.|2001; Marconi \& Hunt 2003; Häring \& Rix 2004; Barth et al. 2005; Lauer et al. 2006; Greene \& Ho 2006; Woo et al. 2006). At masses lower than $\mathcal{M}_{\text {bulge }} \lesssim 10^{10} \mathcal{M}_{\odot}$, the central object with which galaxies correlate may be either intermediate mass BHs in dwarf galaxies (Filippenko \& Ho 2003; Barth et al. 2004, 2005; Greene \& Ho 2006) or central massive star clusters (Ferrarese et al. 2006; Graham \& Driver 2007). The small amount of intrinsic scatter between black holes and bulges is often interpreted to suggest a causal connection between the two - that the growth of one might somehow regulate the other (e.g. Silk \& Rees 1998; Di Matteo et al. 2005).

When did the fundamental BH scaling relationships come about? At higher redshifts, observations are still in the early stages, complicated in part by the challenges of measuring the $\mathrm{BH}$ mass and the bulge properties in the same galaxy. However, recent evidence from the study of quasar host galaxies indicates that a fundamental $\mathcal{M}_{\mathrm{BH}}-\mathcal{M}_{\text {bulge }}$ correlation might have been present as early as $z \sim 4$ (Peng et al. 2006a, b) $)$. Fur-

\footnotetext{
${ }^{1}$ Space Telescope Science Institute, 3700 San Martin Drive, Baltimore, MD 21218; cyp@stsci.edu.

${ }^{2}$ STScI (Giacconi) Fellow
}

thermore, there also appears to be an evolution in the $\mathcal{M}_{\mathrm{BH}}-\mathcal{M}_{\text {bulge }}$ ratio by a factor of $\sim 4$ in the same studies, which points to the possibility that the $\mathrm{BH}$ masses may have matured more quickly than their surrounding stellar bulge mass in the past. In addition, other observations that use [OIII] and $\mathrm{CO}$ emission line widths to infer the gravitational potential of quasar bulges (Shields et al. 2003, 2006; Salviander et al. 2006, 2007; Ho 2007) suggest similar trends ${ }^{3}$, and residual traces of evolution remain detectable even below $z=1$ (Treu et al. 2004, 2007; Woo et al. 2006). Despite the general agreements observationally (but, see Li et al. 2006; Borys et al. 2005), there remain several weaknesses that still complicate the interpretation: a significant factor of 2 systematic uncertainty in the normalization of the $\mathrm{BH}$ mass calibration, the possibility that the normalization of the virial BH mass estimate (Kaspi et al.|2000; Vestergaard 2002; Onken et al.|2004; Kaspi et al.|2005; Greene \& Ho 2005; Peterson \& Bentz 2006; Vestergaard \& Peterson 2006) may evolve with time, because of the fact that the bulge masses are not directly measured, and the possibility that the host galaxy mass may be biased low in high redshift quasars (Lauer et al. 2007) because of the steep decline in the luminosity function of galaxies. With regard to the latter selection bias, it is worthwhile to note, however, that low redshift $(z \lesssim 0.3)$ quasars host galaxies (McLure \& Dunlop 2001), which have similarly high $\mathcal{M}_{\mathrm{BH}}$ and $\mathcal{M}_{\text {gal }}$ to the high redshift objects in Peng et al. (2006a b) , do not exhibit a mass dependent bias even when the scatter is large. Despite these uncertainties, the finding of an existing strong $\mathcal{M}_{\mathrm{BH}}-\mathcal{M}_{\text {bulge }}$ correla-

3 A lack of evolution in the stellar velocity dispersion implies that bulge mass decreases with look-back time (see, e.g, Robertson et al. 2006b). 
tion at $z \gtrsim 1$ is likely to be more secure.

Controversies about the evolution aside, it is not only puzzling that the $\mathcal{M}_{\mathrm{BH}}$-bulge correlations should exist, but that they would have a small intrinsic scatter of 0.3 dex in $\mathcal{M}_{\mathrm{BH}}$ and that the correlation with the bulge mass is practically linear (Marconi \& Hunt 2003; Häring \& Rix 2004; Lauer et al. 2006). These curious facts have received wide theoretical attention over the years and could be explained in a number of ways. For instance, both the $\mathcal{M}_{\mathrm{BH}}-\mathcal{M}_{\text {bulge }}$ and $\mathcal{M}_{\mathrm{BH}}-\sigma_{*}$ relation can be produced by gas accretion onto a nuclear disk (Burkert \& Silk 2001; Cen 2007) or turbulent dissipation of gas (Escala 2006) followed by star formation, by a combination of $\mathrm{BH}$ accretion, galaxy mergers, and star formation (Li et al. 2006; Kauffmann \& Haehnelt 2000; Haehnelt \& Kauffmann 2000), by gravitational collapse of inner parts of a galaxy that forms the bulge from a rotating isothermal sphere (Adams et al. 2001, 2003), by stellar capture (Miralda-Escudé \& Kollmeier 2005) in the accretion disk. The effect of dissipation in galaxy merging on the $\mathrm{BH}$ and fundamental plane scaling relations has also been visited: Ciotti \& van Albada (2001) speculated, and Robertson et al. (2006a) show, that dissipational mergers can produce the $\mathcal{M}_{\mathrm{BH}}-\sigma_{*}$ slope and maintain the fundamental plane of elliptical galaxies. On the other hand, whereas dissipationless mergers of elliptical galaxies also tend to preserve the observed fundamental plane (Boylan-Kolchin et al. 2006; Robertson et al. 2006a), dissipationless mergers of disk galaxies tend to produce a relation that more closely parallels the virial plane.

Despite the aforementioned models, the explanation that has spawned immense activity is the theory of feedback from an active galactic nucleus (AGN). The AGN feedback idea rests observations that quasars typically radiate at a fixed fraction $(10 \%-100 \%)$ of the Eddington luminosity. If such radiation is produced by a massive enough BH, this energy budget is in principle sufficient to quench star formation and terminate the $\mathrm{BH}$ growth itself (e.g. Silk \& Rees 1998; Di Matteo et al. 2005; Cattaneo et al. 2005; Springel et al. 2005; Hopkins et al. 2007, 2006a, and references therein). This idea has been incorporated into cosmological merger simulations (e.g. Granato et al. 2004; Fontanot et al. 2006; Croton et al. 2006), and is seen to have profound possibilities for explaining a wide array of other galaxy evolution puzzles, including the evolution in the galaxy and quasar luminosity functions, mass functions, star formation rates, the X-ray background, and the bimodality of galaxy colors (e.g. see Hopkins et al. 2006a, b, and references therein).

Even though AGN feedback is promising for explaining many aspects of galaxy evolution, it is possible that some other mechanism (or mechanisms) may either have significant influence on the scaling relation between $\mathcal{M}_{\mathrm{BH}}$ and $\mathcal{M}_{\text {bulge }}$, or in fact be more fundamentally the cause. It is therefore worthwhile to look for such factors and to fully study their effects. Dating back years before AGN feedback physics became a popular idea, one such fundamental factor considered by many was galaxy merging (e.g. Kauffmann \& Haehnelt 2000; Haehnelt \& Kauffmann 2000; Ciotti \& van Albada 2001; Nipoti et al. 2003; Islam et al. 2003, 2004; Volonteri et al. 2003). Even without feedback, those simulations already find it possible to produce the $\mathrm{BH}$ scaling relations, albeit to dif- fering degrees of agreement with the observations. However, the reason why a tight linear correlation should emerge is not immediately apparent even when considering no other physics besides merging. In fact, as accorded correctly by intuition, a correlation indeed cannot arise spontaneously from chaotic combinations of galaxies and black hole masses in general. As will be shown below, what does make a tight correlation emerge is the fact that the galaxy mass function decreases with increasing mass. This circumstance brings about a number of interesting implications that will be explored in future studies. In this study, the modest goal is to show that when the focus is switched from the $\mathrm{BH}$-bulge relation, to understanding the more general BH-galaxy relation, some insights may be gained into understanding the growth and evolution of the $\mathcal{M}_{\mathrm{BH}}-\mathcal{M}_{\text {bulge }}$ relation itself.

This study therefore revisits the issue of galaxy merging from the standpoint of a thought experiment. This toy model identifies three basic reasons for why the $\mathcal{M}_{\mathrm{BH}^{-}} \mathcal{M}_{\text {bulge }}$ relation may appear the way it does, even if the $\mathrm{BH}$ masses and their host galaxy masses were completely uncorrelated initially, or if they started out with a steep powerlaw correlation. The over-arching premise is that the galaxy mass function has a Schechter (1976) powerlaw form, so that there is a decline in galaxy number density with increasing mass, especially above masses $\mathcal{M}^{*}$. When this circumstance is met, it can be shown that a "linear attractor" and a central limitlike tendency can work efficiently to produce a linear $\mathcal{M}_{\mathrm{BH}}-\mathcal{M}_{\text {gal }}$ relation, and to reduce the scatter over time. However, while it is tempting to generalize this result to the $\mathcal{M}_{\mathrm{BH}}-\sigma_{*}$ relation, it is not as simple to do because it is not clear how the stellar velocity dispersion scales during galaxy mergers, something that depends on physics not considered in this study (e.g. dissipational vs. dissipationless mergers, star formation, AGN feedback - see Robertson et al. 2006b; Hopkins et al. 2007; Di Matteo et al. 2007; Sijacki et al. 2007).

The main emphasis of the current study is thus only to present a pure statistical exercise, and as such will not invoke merger trees or external physics - besides which many and much more sophisticated models have already been run (e.g. Volonteri et al. 2003; Islam et al. 2004; Granato et al. 2004; Fontanot et al. 2006; Robertson et al. 2006b; Ciotti et al. 2007). In a sense, this work examines a common thread shared by all previous cosmological simulations. While it is tempting to invoke realism by introducing detailed physics from the get-go, e.g. star formation, $\mathrm{BH}$ accretion, and AGN feedback, isolating the effects of simple statistics enables a cleaner exposition of why the convergence behavior should be expected. As such, the current study is not a critique of other, more physical, models which can also explain the same correlation, or to pass critical judgment about which is more or less relevant. Instead, the main message of this study is that, regardless of what other physics may ultimately produce the $\mathcal{M}_{\mathrm{BH}}-\mathcal{M}_{\text {bulge }}$ relation or weaken it, an existing correlation should strengthen if galaxies continue to merge thereafter, whether by major or by minor mergers. In other words, merging alone, in the absence of all other physics, is a sufficient condition to bring about a tight, linear, $\mathcal{M}_{\mathrm{BH}}-\mathcal{M}_{\text {bulge }}$ relation over time, and is always "pulling behind the scenes" to bring about such a corre- 


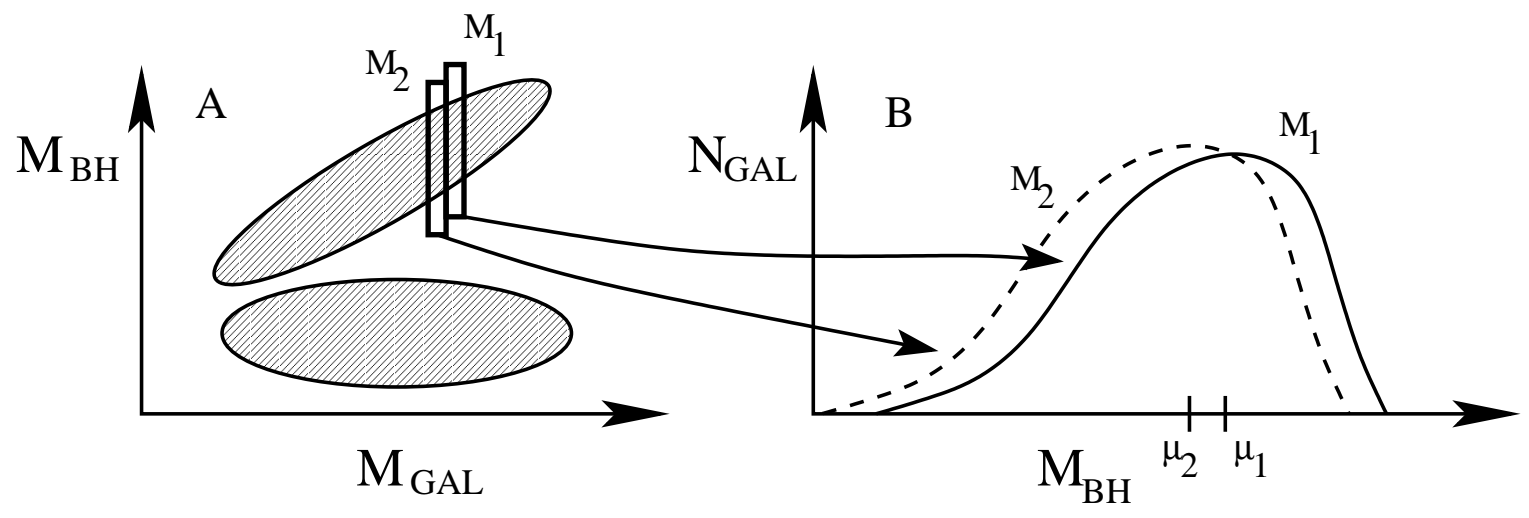

FIg. 1.- (a) Two examples of initial correlations between $\mathcal{M}_{\mathrm{BH}}$ and $\mathcal{M}_{\text {gal }}$. (b) An arbitrary number distribution of $\mathcal{M}_{\mathrm{BH}}$ at each mass slice $\mathcal{M}_{1}$ or $\mathcal{M}_{2}$, where $\mu$ is the mean value of the distribution.

lation. The issue of interest to follow up is to what extent this, or other as yet identified mechanisms, may matter in the end, and to predict more realistic scatter in the $\mathcal{M}_{\mathrm{BH}}-\mathcal{M}_{\text {gal }}$ relation (as opposed to only $\mathcal{M}_{\mathrm{BH}}-\mathcal{M}_{\text {bulge }}$ ) under this hypothesis using more realistic merger trees and physics. We will address this subject in a followup study.

The following discussion will begin by presenting a heuristic view to explain why a linear $\mathcal{M}_{\mathrm{BH}}-\mathcal{M}_{\text {gal }}$ relation is a natural consequence of random merging (Section 2), followed by Monte-Carlo simulations to illustrate the effect (Section 3), and lastly by a discussion and conclusion. In much of the discussion below, the relationship under consideration is more generally the $\mathcal{M}_{\mathrm{BH}^{-}} \mathcal{M}_{\text {gal }}$ relation, whereas $\mathrm{BHs}$ are thought to correlate more tightly with the spheroid component of $\mathcal{M}_{\text {gal }}$, that is $\mathcal{M}_{\text {bulge }}$. It will be seen that the tighter correlation between $\mathcal{M}_{\mathrm{BH}}$ and $\mathcal{M}_{\text {bulge }}$ is a special case of the $\mathcal{M}_{\mathrm{BH}}-\mathcal{M}_{\text {gal }}$ relation and can be understood in the same framework if this hypothesis represents the dominant mechanism by which BHs correlate with galaxy masses.

\section{HEURISTIC PICTURES}

\subsection{The Central Limit Theorem of Galaxy Mergers}

As galaxies undergo merging, it can be shown that the scatter in the $\mathcal{M}_{\mathrm{BH}}-\mathcal{M}_{\text {gal }}$ relation diminishes with increasing number of mergers as a consequence of the central limit theorem (see Appendix). To see this most easily, consider first major mergers, which by definition occur between galaxies of roughly equal mass, often defined to be within the range $\mathcal{M}_{1} / \mathcal{M}_{2} \leq 4$ (Figure 1a). Their similarity in mass also means that the number distribution (i.e. mass function) of $\mathcal{M}_{\mathrm{BH}}$ at a specific galaxy mass, $\mathcal{M}_{1}$, is similar to that at $\mathcal{M}_{2}$, i.e. they are drawn from parent distributions that have roughly the same shape (Figure 1b). However, the mean $(\mu)$ of the distributions might be offset by an amount that depends on the steepness of the $\mathcal{M}_{\mathrm{BH}}-\mathcal{M}_{\text {gal }}$ correlation: the steeper the correlation (Figure 1 $\mathrm{b}$, upper ellipse), the larger is the offset.

Furthermore, consider that in the limit of no initial $\mathcal{M}_{\mathrm{BH}}-\mathcal{M}_{\text {gal }}$ correlation (Figure 1a, lower ellipse), $\mu$ is constant with galaxy mass. The average of any two $B H$ masses randomly drawn from the parent distribution (e.g. Fig. 1b) therefore has a tendency toward the mean value $\mu$ of the parent, by the central limit theorem. This tendency also means that the resulting $\mathrm{BH}$ mass distribution from mergers, obtained by summing BHs drawn from the same distribution, will have a smaller $\log$-normal scatter, $\sigma(\log (\mu))=\sigma(\mu) / \mu$, than the original log-normal distribution, because the fractional mass error, $\sigma\left\langle\mathcal{M}_{\mathrm{BH}, 1}+\mathcal{M}_{\mathrm{BH}, 2}\right\rangle /\left\langle\mathcal{M}_{\mathrm{BH}, 1}+\mathcal{M}_{\mathrm{BH}, 2}\right\rangle$, is smaller, which means the scatter in the $\mathcal{M}_{\mathrm{BH}}$ vs. $\mathcal{M}_{\text {gal }}$ relation, logarithmic on both axes, decreases. In the instance where the initial $\mathcal{M}_{\mathrm{BH}}$ mass distribution (Figure 1b) is normal, with a scatter $\sigma_{\mathrm{BH}, \text { init }}$, the resulting scatter of all galaxies that have undergone one full cycle of major mergers is $\sigma\left(\log \mu_{\mathrm{BH} \text {,merge }}\right)=$ $\sigma\left(\log \mu_{\mathrm{BH}, \text { init }}\right) / \sqrt{2}$ (see Appendix). Therefore an ensemble of galaxies that has undergone $N_{\text {maj }}$ mergers should have a scatter in the $\mathcal{M}_{\mathrm{BH}}-\mathcal{M}_{\text {gal }}$ relation that is reduced by $\sim 2^{0.5 N_{\text {maj }}}$, compared with the initial relation. However, a log-normal $\mathcal{M}_{\mathrm{BH}}$ distribution will have a different convergence rate.

Clearly, this central limit theorem behavior applies to a finite parent distribution of any shape, but the size of the scatter and the rate at which the scatter decreases both depend on the shape of the distribution and the steepness of the initial $\mathcal{M}_{\mathrm{BH}}-\mathcal{M}_{\text {gal }}$ correlation. In the situation where the correlation between $\mathcal{M}_{\mathrm{BH}}$ and $\mathcal{M}_{\text {gal }}$ is steep (e.g. Fig. 1a, upper ellipse) the effective cumulative mass function of $\mathrm{BHs}$ (Figure 1b) residing in galaxies with $\mathcal{M}_{2} \leq \mathcal{M}_{\text {gal }} \leq \mathcal{M}_{1}$ has a wider $\sigma_{\mathrm{BH} \text {,init }}$ than if the $\mathcal{M}_{\mathrm{BH}}-\mathcal{M}_{\text {gal }}$ relation is shallow (Fig. 1ha, lower ellipse). For the same reason, minor mergers also have wider $\sigma_{\mathrm{BH} \text {,init }}$, as the galaxy mass differences are larger, than do major mergers. Therefore, galaxies that have only undergone major mergers would produce an $\mathcal{M}_{\mathrm{BH}^{-}}$ $\mathcal{M}_{\text {gal }}$ relation that is significantly tighter than for galaxies that have only experienced minor mergers, for the same merger rate, and if the initial mass correlation is not flat. This effect is seen in the Monte-Carlo simulations below.

\subsection{Galaxy Merging From a Schechter Mass Distribution}

If the mass density of galaxies follows a Schechter powerlaw form (Schechter 1976),

$$
\Phi(\mathcal{M})=\Phi_{0}\left(\frac{\mathcal{M}}{\mathcal{M}^{*}}\right)^{\alpha+1} \exp \left(-\frac{\mathcal{M}}{\mathcal{M}^{*}}\right)
$$



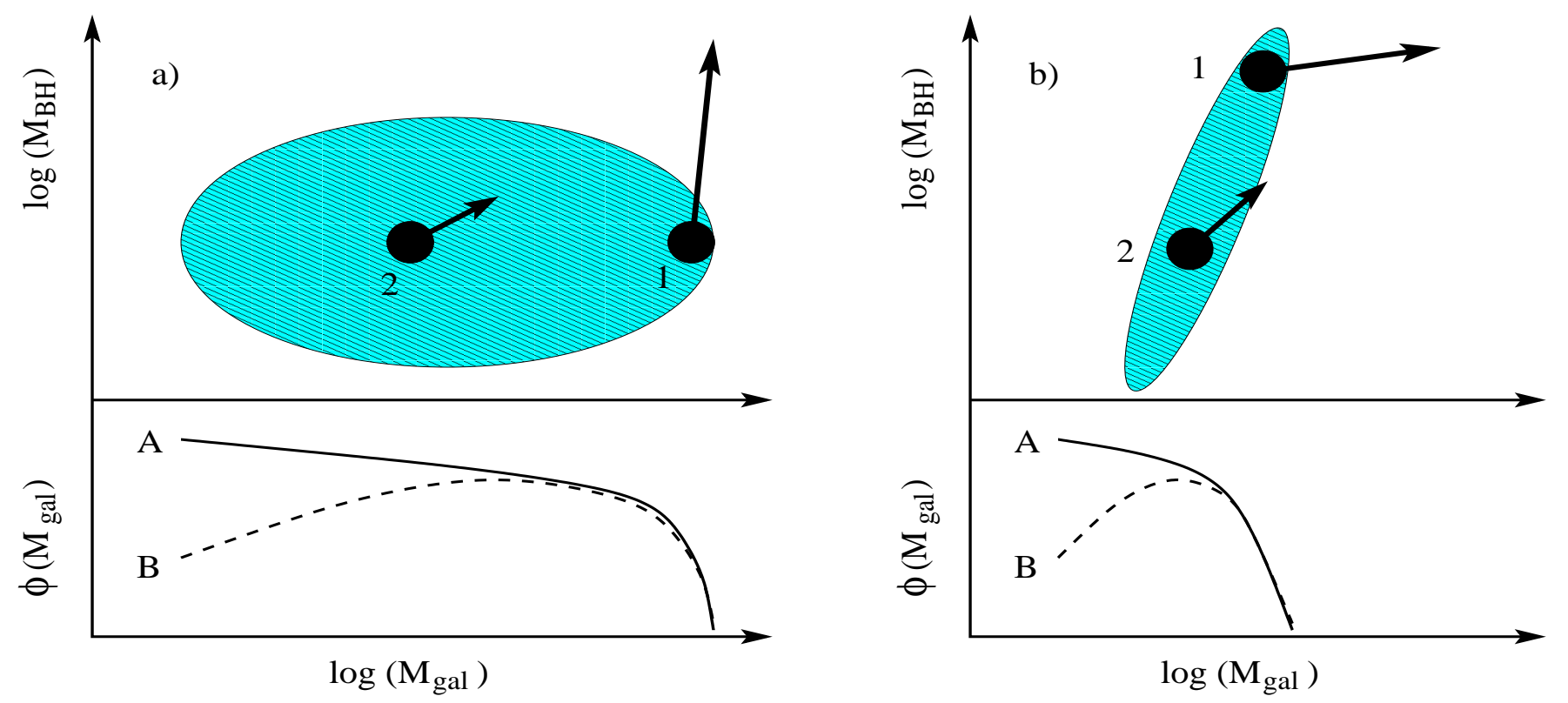

FIG. 2.- (a) No initial correlation in the $\mathcal{M}_{\mathrm{BH}}-\mathcal{M}_{\text {gal }}$ relation. A galaxy at location 1 is more likely to merge with another galaxy at a much lower $\mathcal{M}_{\text {gal }}$ than with one that is comparable to itself (due to the bottom heavy mass function), but roughly of comparable $\mathcal{M}_{\mathrm{BH}}$ (due to non-correlation between $\mathcal{M}_{\mathrm{BH}}-\mathcal{M}_{\text {gal }}$ ). Thus the net evolutionary vector is steep. In contrast, a galaxy at location 2 is likely to merge with another that is comparable in both $\mathcal{M}_{\mathrm{BH}}$ and $\mathcal{M}_{\text {gal }}$ to itself, so the evolution vector is shallower. (b) An initial, strong powerlaw, correlation in the $\mathcal{M}_{\mathrm{BH}}-\mathcal{M}_{\text {gal }}$ relation. A galaxy at location 1 is more likely to merge with another galaxy comparable in $\mathcal{M}_{\text {gal }}$ but at a much lower $\mathcal{M}_{\mathrm{BH}}$ than itself. Thus, the net evolutionary vector is shallow. In contrast, a galaxy at location 2 is likely to merge with another of comparable in $\mathcal{M}_{\mathrm{BH}}$ and $\mathcal{M}_{\text {gal }}$ to itself, so the evolution vector is steeper.

then it is possible also to show that a linear $\mathcal{M}_{\mathrm{BH}}-\mathcal{M}_{\text {gal }}$ relation naturally emerges over time, so that the relation,

$$
\mathcal{M}_{\mathrm{BH}}(z)=\Gamma(z) \mathcal{M}_{\text {gal }}(z)^{\beta}
$$

eventually takes on $\beta=1$. The value of $\Gamma$, which locally is measured to be $\Gamma(0) \sim 1 / 800$ for bulges (e.g. Häring \& Rix 2004), is otherwise arbitrary in the discussion below. $\Gamma$ is degenerate with respect to assumptions about the initial scatter of the $\mathcal{M}_{\mathrm{BH}}-\mathcal{M}_{\text {gal }}$ relation, the initial slope $\beta$, and the initial normalization $\Gamma(\infty)$, for which there are currently insufficient observational constraints; it will not be addressed further in this study. The other assumption used here is that the probability for two galaxies to merge comes from Monte-Carlo sampling of a Schechter mass function (Eq. 1). In actuality, galaxies do not merge randomly, especially at late times. However, complete randomness is only used to facilitate the discussion, and is not a pre-requisite, since the reasoning depends only on the fact that minor mergers occur more frequently than major mergers. This assumption does mean that, depending on the relative balance of major vs. minor mergers, the effects described here, namely convergence toward linearity versus central-limit behavior, may be more relevant at some epoch in time than at others

The reason that a linear correlation emerges through galaxy mergers is illustrated in Figure 2. Figure 2a shows the situation in which the initial $\mathrm{BH}$ and galaxy mass distributions are completely uncorrelated, so that $\beta=0$. The lower part of the diagram shows hypothetical mass functions with two different "faint end" slopes, $A$ and $B$, which will be individually considered in the Monte-Carlo simulation below. If there is no correlation between the $\mathcal{M}_{\mathrm{BH}}$ and $\mathcal{M}_{\text {gal }}$, then the ratio $\mathcal{M}_{\mathrm{BH}} / \mathcal{M}_{\text {gal }}$ will be, on average, larger for low mass galaxies than for high mass galaxies as can be seen by comparing the $\mathcal{M}_{\mathrm{BH}} / \mathcal{M}_{\text {gal }}$ ratio at any two locations, for example, those labeled " 1 " and "2." Therefore, as galaxies merge, a massive object at the extreme end of the mass function, located at position 1 , on average, is more likely to merge with another having a much larger $\mathcal{M}_{\mathrm{BH}} / \mathcal{M}_{\text {gal }}$, thereby evolving the merger product in a steep upward direction, as exaggerated by the vertical arrow. In contrast, an object at position 2 is likely to merge with objects comparable in both $\mathcal{M}_{\mathrm{BH}}$ and $\mathcal{M}_{\text {gal }}$ to itself, so the net evolutionary vector has a shallower slope. Therefore, the cumulative effect of mergers along the mass spectrum is to steepen the massive end of the $\mathcal{M}_{\mathrm{BH}}-\mathcal{M}_{\text {gal }}$ relation relative to the lower extreme, even as the lower end grows in $\mathcal{M}_{\mathrm{BH}}$ and $\mathcal{M}_{\text {gal }}$ on average.

In the other extreme, Figure $2 \mathrm{~b}$, if the primordial relation between the $\mathrm{BH}$ and galaxy masses is steep, corresponding to $\beta \geq 1$, the opposite behavior occurs. Galaxies at location 1 generally have a larger $\mathcal{M}_{\mathrm{BH}} / \mathcal{M}_{\text {gal }}$ ratio than galaxies that have lower mass. Therefore, the $\mathcal{M}_{\mathrm{BH}} / \mathcal{M}_{\text {gal }}$ ratio for massive galaxies would tend to decrease through mergers. The net effect on massive galaxies is to evolve the merger remnant more quickly to the right on average than a lower mass galaxy at location 2 .

Because of a mirror symmetry between Figures 2a and $2 \mathrm{~b}$, the natural equilibrium state of the $\mathcal{M}_{\mathrm{BH}}-\mathcal{M}_{\text {gal }}$ relation is at $\beta=1$, so that further merging of galaxies would evolve remnants along the linear relation, with a constant ratio $\Gamma=\mathcal{M}_{\mathrm{BH}} / \mathcal{M}_{\text {gal }}$. Also because of the con- 
vergence toward this "attractor" state the scatter in the relationship would necessarily decrease over time through galaxy merging.

Lastly, it is worth mentioning that the presence of a break in the galaxy mass function at $\mathcal{M}^{*}$ is a sufficient, but not necessary, condition for convergence toward linearity. A pure powerlaw with $\mathcal{M}^{*}=\infty$ would produce a similar behavior, however, the convergence is slower for flatter slopes $(\alpha \rightarrow-1)$. Furthermore, while the convergence behaviors just described is quite strong for a Schechter mass function with $\alpha=-1$, the convergence would fail for a pure powerlaw with the same slope, because of a lack of a break in the mass function.

\section{MONTE CARLO SIMULATIONS}

To illustrate the idea discussed above, and to quantify how quickly a linear $\mathcal{M}_{\mathrm{BH}}-\mathcal{M}_{\text {gal }}$ relation might emerge, it is useful to consider several numerical simulations for the situations shown in Figure 2. For each of the two scenarios, no-correlation (Fig. 2a) and steep correlation (Fig. 2b), it is also instructive to consider two different initial mass functions, $A$ and $B$, shown in the lower half of Figure 2. The two powerlaw slopes explored below are $\alpha=-1.5$ and $\alpha=-0.5$, respectively. These choices are motivated by observations of the luminosity functions for high redshift galaxies (e.g. Gabasch et al. 2006; Giallongo et al. 2005) under the assumption that light traces mass.

\subsection{Simulation Set-up and Definitions}

Definition of the number of major and minor mergers. - As implied in Section 2, how closely a galaxy lies to the linear part of the $\mathcal{M}_{\mathrm{BH}}-\mathcal{M}_{\text {gal }}$ relation, and how tight the final scatter is for an ensemble of galaxies, will depend on the cumulative merger history. Therefore, the most useful way to understand the simulation results is to define the number of major mergers, $N_{\text {maj }}$, as the cumulative sum of all such events over the entire tree for a given galaxy, not just in the most immediate, that is, the main, branch. For example, even if a galaxy has never experienced a single major merger on the main branch in its lifetime, it could still lie close to the final, linear part of the $\mathcal{M}_{\mathrm{BH}}-\mathcal{M}_{\text {gal }}$ relation because the progenitors of the main branch, and their progenitors, and so forth, could have experienced a number of major mergers.

Evolution of the mass function. - One issue to consider is how the galaxy mass function might evolve, and whether the path of evolution might affect the final convergence. The effect of galaxy masses growing with time is to both increase $\mathcal{M}^{*}$ and steepen the "faint end slope" $(\alpha)$ of the Schechter function. As galaxy populations grow in mass and number density, the rate of change in $\mathcal{M}^{*}$ and $\alpha$ would affect the rate of convergence to the final $\mathcal{M}_{\mathrm{BH}}-\mathcal{M}_{\text {gal }}$ normalization, slope, and scatter. Predicting the rate of change in the $\mathcal{M}_{\mathrm{BH}}-\mathcal{M}_{\text {gal }}$ relation requires realistic merger trees and accounting for other detailed physics such as feedback, which will be addressed in a followup study. For the current purposes of showing that convergence toward a linear $\mathcal{M}_{\mathrm{BH}}-\mathcal{M}_{\text {gal }}$ relation does naturally occur, it suffices to consider two scenarios: a replenishment scenario, in which the Schechter mass function is continuously, and randomly, replenished as galaxies merge, and a depletion scenario, in which no new galaxies are formed to take the place of those that have merged. Combined with considerations about the initial mass function slopes, the simulations will have covered the gamut of sensible possibilities and conditions that may be present at early and late cosmic epochs.

Initial scatter of the $\mathcal{M}_{\mathrm{BH}}-\mathcal{M}_{\text {gal }}$ relation and the initial mass function $\mathcal{M}^{*}$. - In the simulation, the distribution of galaxies is first drawn randomly from the Schechter mass function, after which a BH mass is assigned, following Equation 2, by drawing from a log-normal Gaussian distribution with a generous Gaussian dispersion of $\sigma_{\mathrm{BH}}=2$ dex, i.e. a scatter of a factor 100 in mass. The exact choice of the initial scatter is directly proportional to, but otherwise only partially determines, the final scatter in the $\mathcal{M}_{\mathrm{BH}}-\mathcal{M}_{\text {gal }}$ relation. Other factors that determine the final scatter depend on how long the simulation runs, and on the initial value of $\mathcal{M}^{*}$. Currently, there are some observational constraint on the rate of mergers, which will be considered in a followup study. In this study, the results are merely normalized arbitrarily to match the final $\mathcal{M}_{\mathrm{BH}}-\mathcal{M}_{\text {gal }}$ relation observed today.

The simulation "clock." - The progress of time is not well defined in Monte-Carlo simulations, so it is useful to define merging cycles for the sake of keeping track of the simulation progress. Each full cycle is defined as being complete after the number of merger events equals the number of galaxies present at the beginning of that particular cycle. Galaxies that are produced or merged retain their states for the following cycle. Because some galaxies may merge multiple times by being drawn repeatedly, not all galaxies will be involved in mergers after each full cycle. The exact definition of the simulation clock is unimportant, as it is only the relative number of major vs. minor mergers on average that determines the degree of convergence toward a linear $\mathcal{M}_{\mathrm{BH}}-\mathcal{M}_{\text {gal }}$ relation, where a major merger is defined as having a mass ratio of at most 4:1.

\subsection{Replenishment Scenarios}

The replenishment scenario is one of the two simple ways considered to emulate the progress of galaxy evolution. Here, by definition, the rate of galaxy mergers equals the rate of galaxy number production. The way a galaxy is newly produced is by being selected randomly from an initial mass function, parameterized by $\alpha$ and $\mathcal{M}^{*}$, which does not evolve with time. In contrast, galaxies "grow" only by merging with another member in the galaxy pool existing at the time, and hierarchical merging is the only avenue for mass growth. Therefore, as galaxies merge, the cumulative mass function does undergo evolution. However, the total number of galaxies remains constant because of the 1:1 ratio of merging:replenishment.

Figures 3 and 4 illustrate the results for initial conditions $\beta=0$ (i.e. no $\mathcal{M}_{\mathrm{BH}}-\mathcal{M}_{\text {gal }}$ correlation) and $\beta=2$ (steep $\mathcal{M}_{\mathrm{BH}}-\mathcal{M}_{\text {gal }}$ correlation), respectively, showing only a small subset of the data points. In each figure, two different initial mass functions, $\alpha=-0.5$ (Figs. 3a, $4 a$ ) and $\alpha=-1.5$ (Figs. 3b, 4b) are considered. In each of the Figures, the initial distribution of the $\mathcal{M}_{\mathrm{BH}^{-}} \mathcal{M}_{\text {gal }}$ relation (or lack thereof) is shown with crosses. The open colored data points show the $\mathcal{M}_{\mathrm{BH}}-\mathcal{M}_{\text {gal }}$ development of galaxies that have undergone $N_{m a j} \geq 5$ major merger episodes, after 10 (blue triangles), 100 (green squares), 

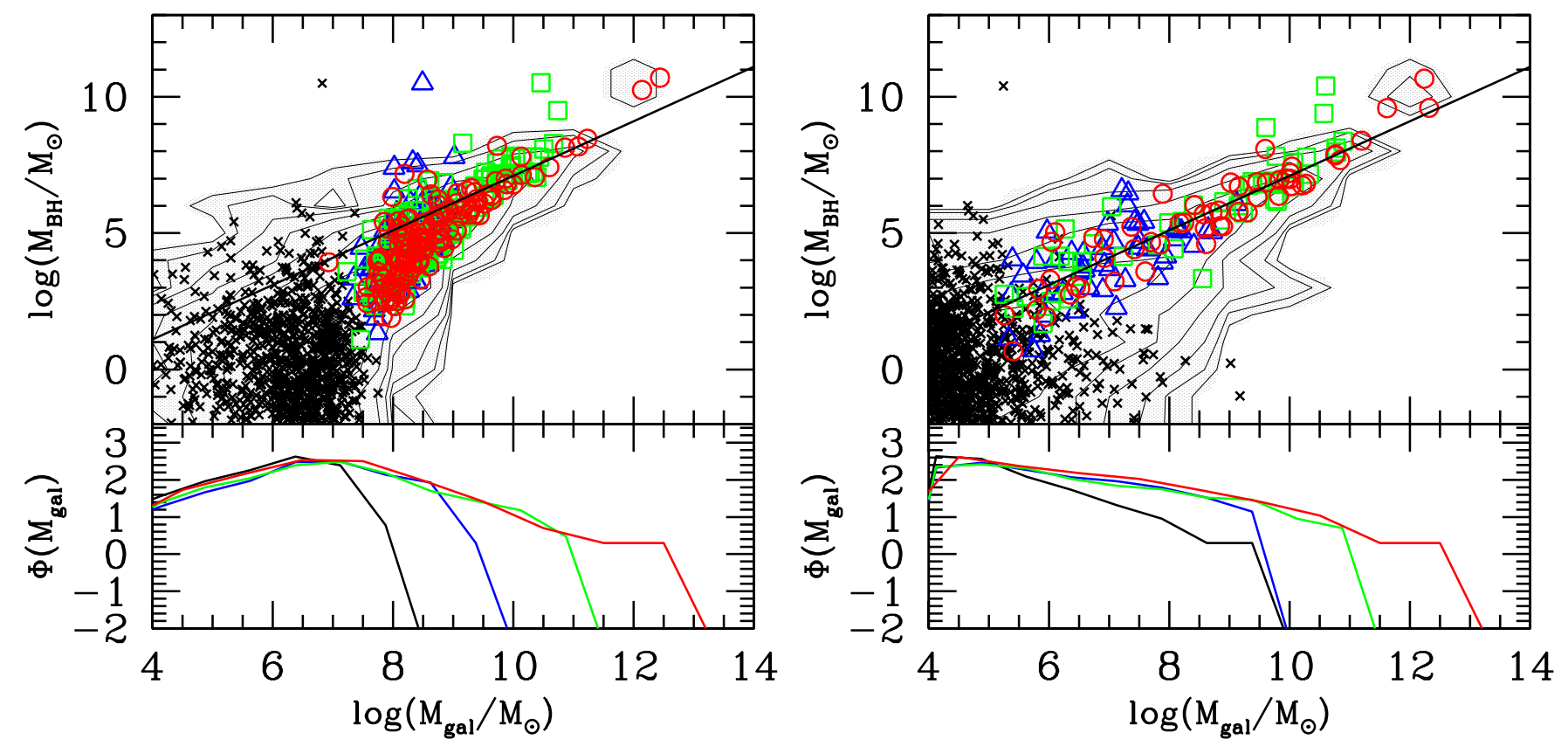

FIg. 3.- No initial correlation $(\beta=0)$ in the $\mathcal{M}_{\mathrm{BH}}-\mathcal{M}_{\text {gal }}$ relation, in the replenishment scenario. The black crosses represent the initial distribution of points, and the solid line shows the local $\mathcal{M}_{\mathrm{BH}}-\mathcal{M}_{\text {gal }}$ relationship from Häring \& Rix (2004) - it is not a fit to the data points. The colored data points represent objects that have undergone at least five major merger episodes after 10 (blue triangles), 100 (green squares), and 1000 (red circles) complete "merging cycles." The crosses are the primordial distribution, corresponding to the initial mass function. The shaded region illustrates the locus of all points after 1000 cycles; the density of points doubles with each contour level. The cumulative histograms after the corresponding merger sequences are shown below the data points. (a) An initial Schechter powerlaw slope of $\alpha=-0.5$. (b) An initial Schechter powerlaw slope of $\alpha=-1.5$.
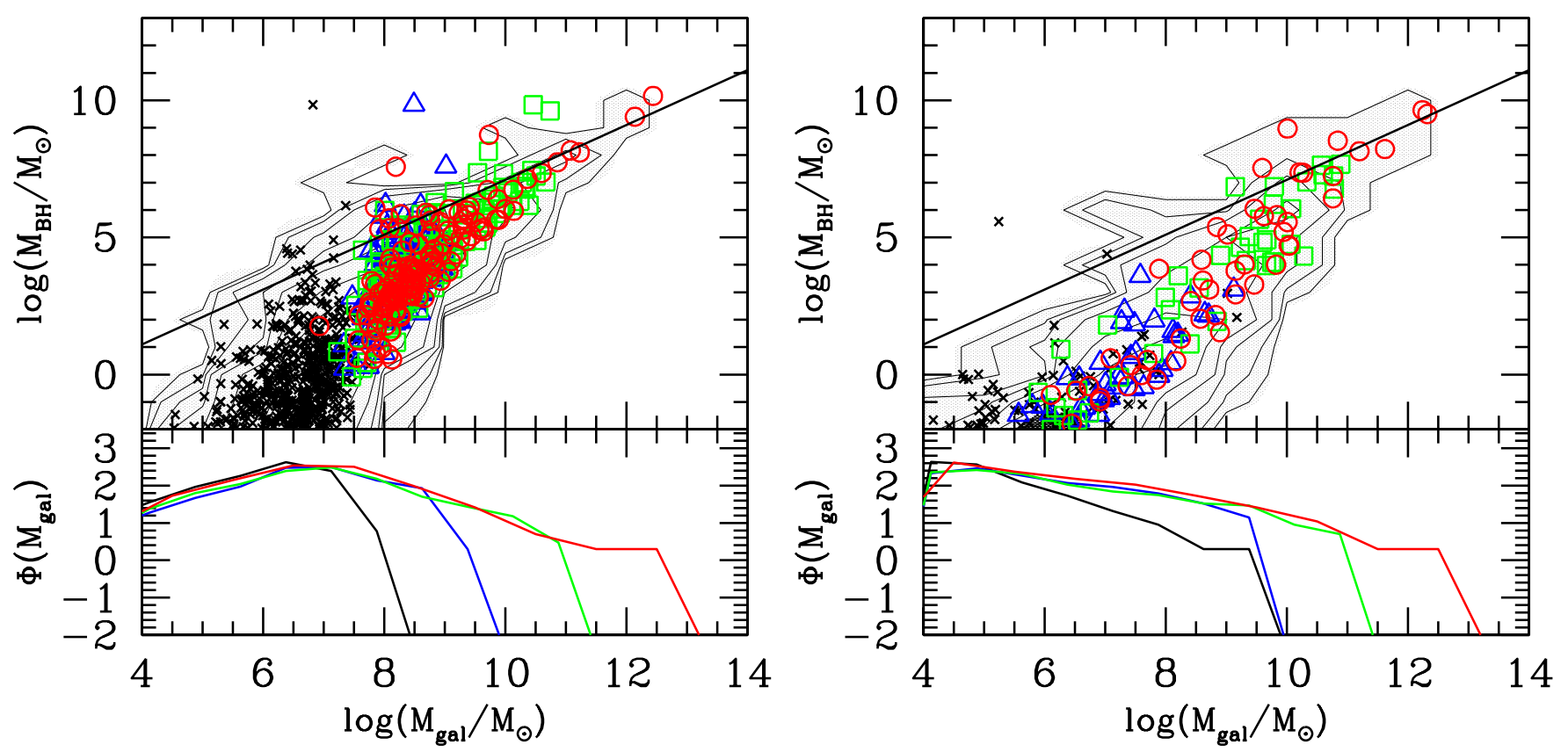

FIG. 4.- Similar to Figure 3, except for a steep initial correlation $(\beta=2)$ in the $\mathcal{M}_{\mathrm{BH}}-\mathcal{M}_{\text {gal }}$ relation. See Figure 3 for details. 


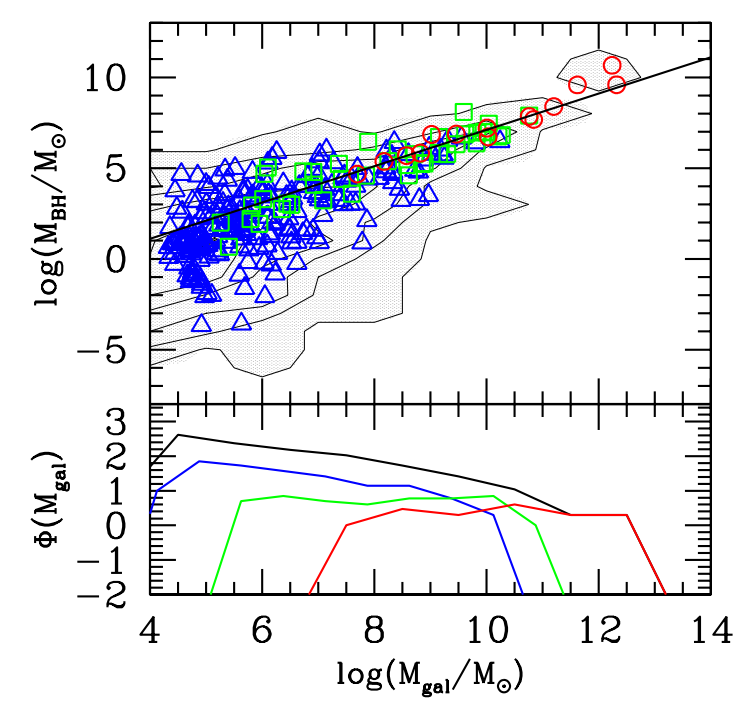

FIG. 5.- Similar to Figure 3b, showing the effect of central-limit tendencies with increasing number of major mergers for galaxies after 1000 merger cycles. The colored data points illustrate objects that have undergone $1 \leq N_{\text {maj }} \leq 4$, (blue triangles), $5 \leq N_{\text {maj }} \leq$ 14 (green squares), $N_{\text {maj }}>14$ (red circles) major mergers. The greyscale contours shows the locus of all the points.

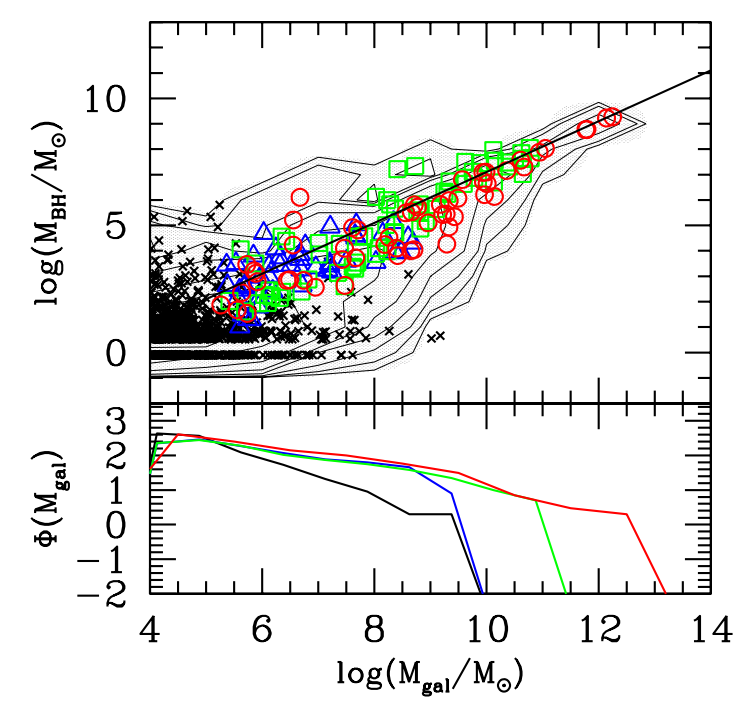

FIG. 6.- Similar to Figure 3, except the BH mass is drawn from a Schechter law of $\alpha=-1.5$ instead of a Gaussian distribution. See Figure 3 for details.

and 1000 (red circles) merger cycles have transpired. These data points effectively illustrate the progress of the $\mathcal{M}_{\mathrm{BH}}-\mathcal{M}_{\text {gal }}$ evolution for objects that might be morphologically identified as early-type galaxies of each cycle. For clarity, the contour levels represent the locus of points after 1000 merger cycles, and the levels are spaced at multiples of 2 in density. The luminosity functions of the galaxy pool at the end of the merger cycles are shown in the lower half of each diagram in corresponding colors and locale in mass. Lastly, a linear reference line is overplotted in the Figures with normalization given by $\mathcal{R}_{0}=800$ (Häring \& Rix 2004), and the simulations are scaled/shifted arbitrarily to match; it is not a fit to the data points.

As shown in Figures 3 and 4, the convergence towards a tight linear relation is fairly quick. After five major merger episodes a linear relation starts to emerge regardless of the initial conditions of the mass function or the form of the $\mathcal{M}_{\mathrm{BH}}-\mathcal{M}_{\text {gal }}$ correlation. One reason for this quick convergence is the central-limit behavior of major mergers which is shown in Figure 5, in which the increasing number of mergers is represented by different symbols and shades. The one notable case where the convergence toward linearity is slower than the other scenarios is Figure $4 b$, where the effect is only evident at $10^{10.5} \mathcal{M}_{\odot}$ or greater, even as the scatter has decreased markedly. In general, if the $\mathcal{M}_{\mathrm{BH}}-\mathcal{M}_{\text {gal }}$ correlation is steep initially, the tail at low mass remains steep after a large number of major mergers has occurred, even as the massive end converges toward linearity.

Lastly, the qualitative convergence effects do not depend on the assumption about the distribution of $\mathrm{BH}$ mass at each galaxy mass. Figure 6 shows an example that is in direct analog to Figure 3b, except that the $\mathrm{BH}$ mass is instead drawn from a Schechter mass function with $\alpha=-1.5$.

\subsection{Depletion Scenarios}

The other extreme of the merger simulations is to consider what effect galaxy depletion from a finite reservoir has on the $\mathcal{M}_{\mathrm{BH}}-\mathcal{M}_{\text {gal }}$ relation. Because the number density of galaxies builds up over time, the depletion scenario is expected to not be realistic. Nevertheless, it is useful for illustrating how the $\mathcal{M}_{\mathrm{BH}}-\mathcal{M}_{\text {gal }}$ convergence is affected by a different evolution in the mass function as compared with the replenishment scenario.

The depletion scenarios are constructed by creating a large sample of $5 \times 10^{5}$ objects, initially having no correlation between $\mathrm{BH}$ and galaxy masses (Figure 7) or with a $\beta=2$ correlation between the two (Figure 8). The $\mathrm{BH}$ masses are assigned to the galaxies with a log-normal distribution of dispersion $\sigma=2$ centered around Equation 2. In each scenario, galaxies are created to have initial mass functions of $\alpha=-0.5$ (Figures 7a and 8a) or $\alpha=-1.5$ (Figures $7 \mathrm{~b}, 8 \mathrm{~b}$ ). Then, as galaxies merge, no new ones are created to replace them. As a consequence, the mass function evolves by growing in $\mathcal{M}^{*}$, the number density decreases, and a sharp truncation develops at low masses (see lower half of Figures 7 and 8). As the number of merging cycles increases, the scatter decreases quickly and converges toward a linear relation, as illustrated by the solid line. Once again, as shown in Figure 8 (especially $8 \mathrm{~b}$ ), the convergence is much slower for steep $\alpha$ and steep $\beta$ compared with other scenarios. And while the convergence trends are noticeable, because of a dearth of minor galaxies with which to merge at late times (red circles), the slope is virtually "frozen in," and the subsequent convergence is due mostly to the centrallimit theorem.

\section{DISCUSSION AND CONCLUSION}

This study has revisited the issue of how galaxy merging may affect the $\mathcal{M}_{\mathrm{BH}}-\mathcal{M}_{\text {gal }}$ scaling relation from the standpoint of basic mass addition and statistics, thereby clearly isolating the merger cause from other de- 

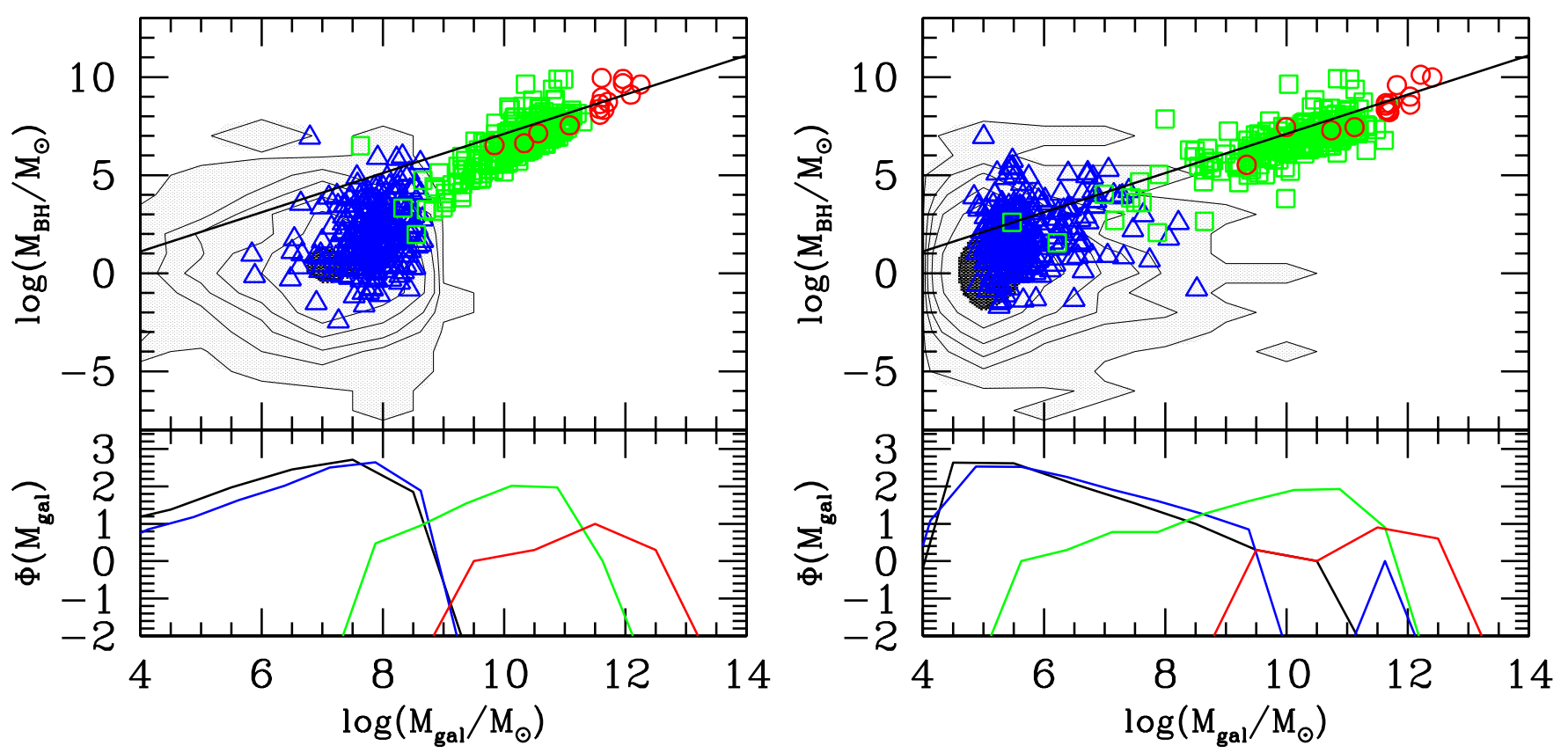

FIG. 7.- No initial correlation $(\beta=0)$ in the $\mathcal{M}_{\mathrm{BH}}-\mathcal{M}_{\text {gal }}$ relation, depletion scenario. The contours represent the initial distribution of points, and a solid line shows the local $\mathcal{M}_{\mathrm{BH}}-\mathcal{M}_{\text {gal }}$ relationship from Häring \& Rix (2004) - it is not a fit to the data points. The colored data points represent objects that have undergone at least 1 major merger episodes after 1 complete merging cycles (blue triangles), 10 (green squares), and 14 (red circles). The cumulative histograms after the corresponding merger sequences are shown below the data points. a) An initial Schechter powerlaw slope of $\alpha=-0.5$. b) An initial Schechter powerlaw slope of $\alpha=-1.5$.
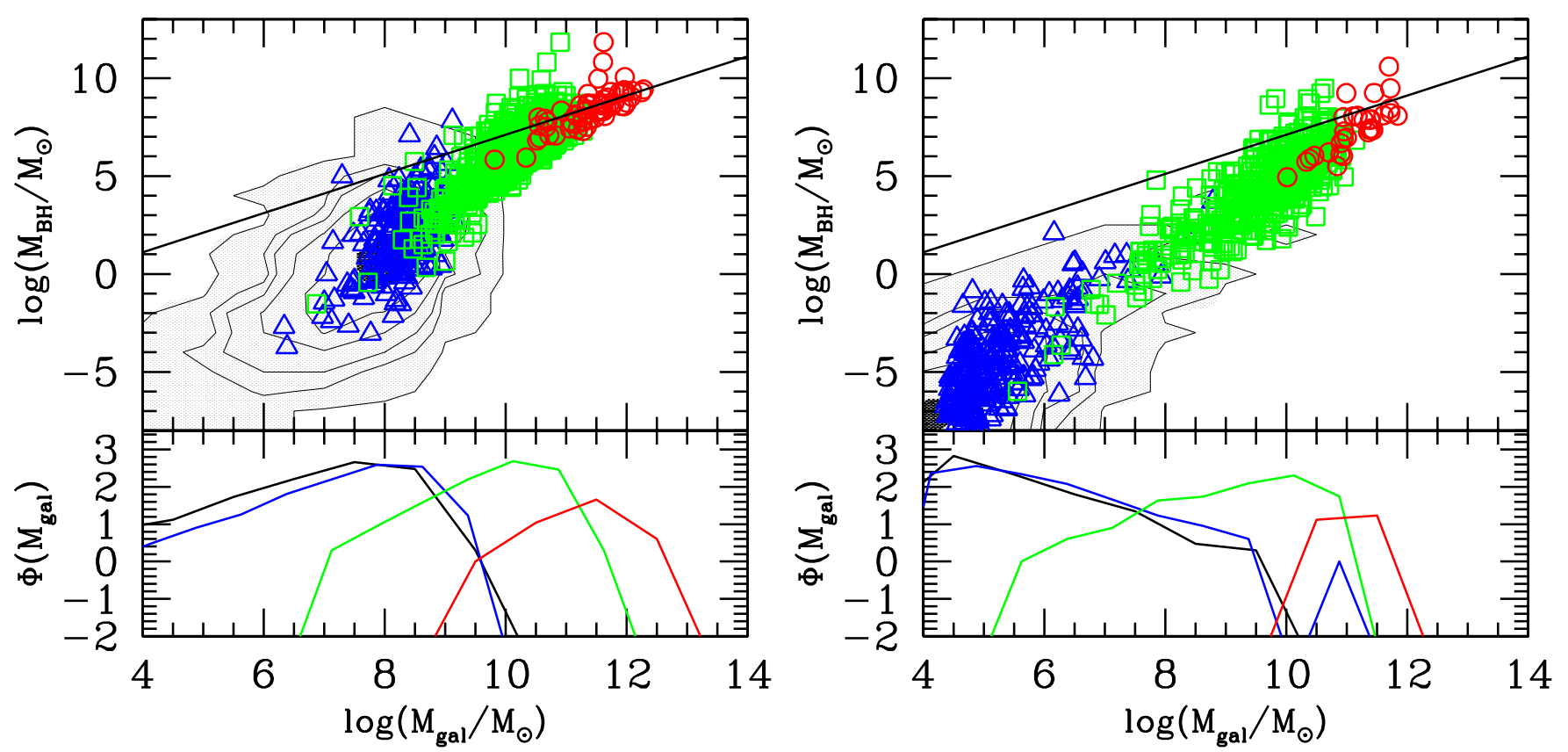

FIG. 8.- Similar to Figure 7, except for a steep initial correlation $(\beta=2)$ in the $\mathcal{M}_{\mathrm{BH}}-\mathcal{M}_{\text {gal }}$ relation. See Figure 7 for details. 
tailed physics that must otherwise affect galaxy evolution. Through Monte-Carlo simulations, a tight, linear, $\mathcal{M}_{\mathrm{BH}^{-}} \mathcal{M}_{\text {gal }}$ correlation appears to emerge when galaxies have undergone five or more major mergers (along the entire tree, not just the main branch), and many minor ones, for practically all reasonable initial correlations between $\mathcal{M}_{\mathrm{BH}}$ and $\mathcal{M}_{\text {gal }}$, or a lack of one. The main reasons for these behaviors are seen to be the following:

1. The galaxy mass function decreases with increasing mass.

2. Major mergers have a strong central-limit tendency, so that regardless of the initial $\mathcal{M}_{\mathrm{BH}^{-}} \mathcal{M}_{\text {gal }}$ correlation, the scatter should decrease with an increasing number of events. While this tendency also acts on minor mergers, the drive toward smaller scatter is weaker because minor mergers occur between galaxies that are vastly discrepant in both galaxy and $\mathrm{BH}$ mass as compared with major mergers, by definition. The corollary is that the steeper a correlation between $\mathcal{M}_{\mathrm{BH}}(y$-axis) and $\mathcal{M}_{\text {gal }}$, the stronger the central-limit tendency for major mergers compared with minor. However, major mergers alone are not enough to cause the $\mathcal{M}_{\mathrm{BH}}-\mathcal{M}_{\text {gal }}$ relation to converge to linearity over time because the ratio $\mathcal{M}_{\mathrm{BH}} / \mathcal{M}_{\text {gal }}$ is not changed much.

3. Minor mergers are primarily responsible for causing the $\mathcal{M}_{\mathrm{BH}}-\mathcal{M}_{\text {gal }}$ relation to converge toward a linear — that is, $\mathcal{M}_{\mathrm{BH}}=\Gamma \mathcal{M}_{\text {gal }}$ - relation because the mass function of galaxies follows a Schechter powerlaw. Without minor mergers, the $\mathcal{M}_{\mathrm{BH}}-\mathcal{M}_{\text {bulge }}$ relation can be "frozen" to a slope that is not necessarily linear. This linear attractor causes a convergence toward a tighter $\mathcal{M}_{\mathrm{BH}}-\mathcal{M}_{\text {gal }}$ relation; however, it is less efficient at reducing the scatter compared with the central-limit seeking tendency of major mergers, as shown in Figure 5.

It is curious that galaxy merging itself might produce a linear $\mathcal{M}_{\mathrm{BH}}-\mathcal{M}_{\text {gal }}$ relation. However, a natural question that does arise is, "When is the merging statistics presented in this study relevant?" On the surface, it is easy to conclude that because the reasoning refers to a two component model it ought to apply to "dry" mergers, but perhaps not to a three component model involving stars, gas, and $\mathrm{BH}$. Thus, the implication is also that it ought not apply to galaxies undergoing gasrich mergers, that is, early cosmic history. However, it is not clear that such a skepticism is warranted. For example, in the entire discussion thus far, the abscissa, $\mathcal{M}_{\text {gal }}$, might just as well refer to $\mathcal{M}_{\text {gal }}=\mathcal{M}_{\text {stellar }}+\mathcal{M}_{\text {gas }}$, instead of just $\mathcal{M}_{\text {stellar. }}$. If BHs do not grow much by accretion and that the gas does not get removed from the definition of $\mathcal{M}_{\text {gal }}$ during mergers, then the $\mathcal{M}_{\mathrm{BH}^{-}}$ $\mathcal{M}_{\text {gal }}$ correlation can emerge from statistical merging. The argument holds true even if $\mathcal{M}_{\text {gas }}$ transforms arbitrarily into $\mathcal{M}_{\text {stellar }}$, as long as the sum is conserved. In the limit where BHs do grow most of their mass during AGN accretion, as might be implied by Soltan (e.g. 1982); Yu \& Tremaine (e.g. 2002), so that $\Delta \mathcal{M}_{\mathrm{BH}} \propto \mathcal{M}_{\text {gas }}$ and $\Delta \mathcal{M}_{\mathrm{BH}} \gg \mathcal{M}_{\mathrm{BH}}$, then the correlation between $\mathcal{M}_{\mathrm{BH}}$ and $\mathcal{M}_{\text {gal }}$ comes out by construction rather than by statistical merging. However, statistical reasoning would still be a "supporting actor" to reduce the scatter and to forcibly steer the $\mathcal{M}_{\mathrm{BH}}-\mathcal{M}_{\text {gal }}$ relation in the preferred linear direction. Likewise, even if $\mathrm{BH}$ growth, or other physics (e.g. gravitational radiation, three body $\mathrm{BH}$ ejection - Merritt et al. 2004; Volonteri \& Perna 2005; Ciotti et al. 2007, and references therein), were a "heating" source, that is, one that randomizes a tight linear $\mathcal{M}_{\mathrm{BH}}-\mathcal{M}_{\text {bulge }}$ relation, the linear and central limit attractors would cause a re-convergence if galaxies continue to merge thereafter by both major and minor mergers. In summary, while it is entirely possible that the $\mathcal{M}_{\mathrm{BH}^{-}}$ $\mathcal{M}_{\text {bulge }}$ relation has origins outside of basic statistics, galaxy merger statistics can still affect the final outcome of a $\mathcal{M}_{\mathrm{BH}}-\mathcal{M}_{\text {gal }}$ correlation in both the scatter and the slope. In any event, statistical reasoning is a fundamentally robust explanation for why random galaxy merging does not corrupt a pre-existing $\mathcal{M}_{\mathrm{BH}}-\mathcal{M}_{\text {bulge }}$ relation, which is important to bear in mind in the context of the $\mathcal{M}_{\mathrm{BH}}-\mathcal{M}_{\text {bulge }}$ or $\mathcal{M}_{\mathrm{BH}}-\mathcal{M}_{\text {gal }}$ relation in a hierarchically forming universe.

While the $\mathcal{M}_{\mathrm{BH}}-\mathcal{M}_{\text {bulge }}$ relation might have other origins, it is nonetheless interesting and revealing to follow through the consequences of statistical merging. For instance, simple statistics naturally explains why black holes appear to correlate most strongly with galaxy bulges, rather than more generally with a galaxy as a whole, which might include a stellar disk (Kormendy \& Gebhardt 2001): bulge masses, assuming they were assembled through major mergers, have a stronger central-seeking tendency than disk galaxies, whose growth history might involve more minor merger events. As such, the $\mathcal{M}_{\mathrm{BH}}-\mathcal{M}_{\text {bulge }}$ relation is a special case of a more fundamental $\mathcal{M}_{\mathrm{BH}}-\mathcal{M}_{\text {gal }}$ relation. Reversing the argument, the observational fact that $\mathcal{M}_{\mathrm{BH}}$ correlates most strongly with bulge masses, coupled with the central-limit theorem reasoning, implies that the merger trees of elliptical galaxies were more dominated by major merger events than were disk galaxies. Conversely, the fact that the scatter in the $\mathcal{M}_{\mathrm{BH}^{-}} \mathcal{M}_{\text {gal }}$ relation is observed to be much larger for disk dominated galaxies implies, statistically, that their progenitors, and progenitors thereof, have undergone more minor mergers.

The possibility that a more fundamental correlation is between $\mathcal{M}_{\mathrm{BH}}$ and $\mathcal{M}_{\text {gal }}$ (rather than $\mathcal{M}_{\text {bulge }}$ ) also has practical implications for what slope and scatter would be measured by observations. First, because the slope changes with mass even for objects that experienced the same number of major mergers (e.g. Figures 3 and 7), the deviation from linearity and the intrinsic scatter will depend exactly on how the data are cut. Simply defining a sample of objects based on a mass selection cut will bias one's measurement of the slope and scatter. Furthermore, defining a sample based on morphology criteria may also implicitly preselect samples that have certain major vs. minor merger histories. Observationally, it is therefore crucial, when comparing intrinsic scatter and slope of the $\mathcal{M}_{\mathrm{BH}}-\mathcal{M}_{\text {bulge }}$ relation to be specific about sample selection parameter space, morphology, bulgeto-disk ratios, or other criteria, lest the conclusions be caused by subtle but trivial selection biases.

Another consequence of this thought experiment is that the ratio $\Gamma=\mathcal{M}_{\mathrm{BH}} / \mathcal{M}_{\text {bulge }}$ approaches an asymp- 
totic value with time from having a smaller ratio in the past. On the surface, this appears contrary to the findings of Peng et al. (2006a b) ; Woo et al. (2006); Shields et al. (2003, 2006) based on quasar host galaxy studies that the ratio $\Gamma$ decreases over time. If the quasar host galaxy studies are correct and are not significantly affected by biases pointed out by Lauer et al. (2007), then some other physics not considered here is responsible for causing a decline in the normalization of $\Gamma$ with time (e.g. see Croton 2006; Hopkins et al. 2007; Fontanot et al. 2006). For instance, the abscissa is ambiguous about what mass $\mathcal{M}_{\text {gal }}$ corresponds. If gas mass is a significant fraction of a galaxy's mass, then forming stars out of the gas reservoir would decrease $\Gamma$ over time, if the abscissa $\mathcal{M}_{\text {gal }}$ represents the galaxy's stellar bulge mass. Secular growth of galaxy bulges by accreting stars in galaxy disks would also decrease $\Gamma$, at the expense of increasing the scatter. Major mergers of pure stellar bulges, however, would not cause $\Gamma$ to decrease over time.

In hindsight, the results of this study could have been anticipated from Islam et al. (2003, 2004); Ciotti et al. (2007), given that the initial conditions used in those studies are a special case of this one where the initial $\mathrm{BH}$ scatter $\sigma_{\mathrm{BH}} \rightarrow 0$ (Figure $3 \mathrm{a}$ or $3 \mathrm{~b}$ ) (M. Volonteri and L. Ciotti 2007, private communication). Just as relevant, Croton (2006); Ciotti et al. (2007) show that once the $\mathcal{M}_{\mathrm{BH}}-\mathcal{M}_{\text {bulge }}$ relation is in place, it is fairly impervious to being randomized by galaxy merging. And because Islam et al. (2003) also uses realistic cosmological merger trees, they confirm that the arguments presented here ought to remain relevant. However, the reasons behind the $\mathcal{M}_{\mathrm{BH}}-\mathcal{M}_{\text {bulge }}$ convergence behavior are difficult to extract from previous studies because of the use of priors, the use of identical BH seeds, the inclusion of other physics, and the focus on only the BH-bulge coevolution (i.e. major mergers). The latter, especially, is worth examining further, because the prior that one chooses about whether the $\mathrm{BH}$ correlates with just its bulge or with the entire galaxy can lead to differing interpretations.

In particular, one conclusion from Islam et al. (2003, 2004) is that the $\mathcal{M}_{\mathrm{BH}}-\mathcal{M}_{\text {bulge }}$ relation converges to a slightly non-linear slope of $\beta=0.9$; hence they reason that other physics, perhaps $\mathrm{BH}$ growth through accretion of gas, is required in order to increase the slope closer to linearity. The reasoning presented in the current study, however, would stipulate that linearity is an asymptotic outcome of mergers, but deviations from lin- earity come from the possibility that the low mass galaxies have not yet achieved the asymptotic limit, because of a weaker convergence. At low masses, the slope deviates from unity in either direction depending on the initial mass function of the galaxies (e.g. compare Figure $3 \mathrm{a}$ with $3 \mathrm{~b}$ ), on the mass cut of the study, and on the relative incidence of minor versus major mergers.

An interesting consequence to consider is how the $\mathcal{M}_{\mathrm{BH}^{-}} \mathcal{M}_{\text {gal }}$ relation might differ between high and low density galaxy clusters. However, one of the unrealistic side-effects of using Monte-Carlo simulations to determine merger rates is that the normalization of the $\mathcal{M}_{\mathrm{BH}}-\mathcal{M}_{\text {bulge }}$ relation is the same in all density regimes. This is because the normalization factor, $\Gamma$, depends only on the ratio of major to minor merger events which, in the Monte-Carlo universe, is not affected by a simple rescaling of the mass function. However, in the real universe, the relative rates of major and minor mergers can change with density and, as such, may result in different normalization and scatter in the $\mathcal{M}_{\mathrm{BH}}-\mathcal{M}_{\text {gal }}$ relation.

Lastly, because of the ambiguity in what $\mathcal{M}_{\text {gal }}$ corresponds, depending on whether it refers to the total stellar mass, gas mass, dark matter halo mass, or a combination thereof, the degree of scatter and linearity would clearly differ, as a result of different initial mass functions and merger histories. Because the scenarios considered above depend on a linear addition of masses, the arguments therefore may not apply to gas masses that are not gravitationally bound to a galaxy. These and other issues will be addressed in a future study, which will incorporate the use of realistic merger trees.

\section{ACKNOWLEDGMENT}

Over the course of this study, I have greatly enjoyed lively discussions with many friends and colleagues, including Jenny Greene, Hans-Walter Rix, Luis Ho, Eric Bell, Michael Santos, Robert Kennicutt, Rachel Somerville, Aaron Barth, Christy Tremonti, Darren Croton, Tommaso Treu, and David Koo. I also thank Jim Rose, Wayne Christiansen, Marta Volonteri, Luca Ciotti, and Alister Graham for comments and discussions on past and future studies. This study greatly benefited from discussions with Phil Hopkins on issues related to AGN feedback over the past several months. I also gratefully acknowledge insightful comments and suggestions from the referee, and Science Editor Chung-Pei Ma, and the support of STScI through the Institute (Giacconi) Fellowship Program.

\section{APPENDIX}

This appendix shows that the $\mathcal{M}_{\mathrm{BH}}-\mathcal{M}_{\text {bulge }}$ relation follows a central-limit-like behavior when galaxies undergo major mergers. Specifically, this means that if the initial parent distribution of progenitor $\mathcal{M}_{\mathrm{BH}}$ that undergoes merging is, for simplicity, normally distributed about a mean BH mass $\mu$, thus having a logarithmic dispersion $\sigma\left(\log \left(\mu_{\text {init }}\right)\right)$, then a new distribution of $\mathcal{M}_{\mathrm{BH}} \mathrm{s}$ after merging will have a log-normal dispersion that scales as: $\sigma\left(\log \left(\mu_{\text {merge }}\right)\right) \sim \sigma\left(\log \left(\mu_{\text {init }}\right)\right) / \sqrt{2}$

First, the mean, $\mu_{\text {merge }}$, of the resulting $\mathrm{BH}$ distribution after two BHs merge from the initial parent distribution is:

$$
\mu_{\text {merge }}=\left\langle\mathcal{M}_{\mathrm{BH}, 1}+\mathcal{M}_{\mathrm{BH}, 2}\right\rangle,
$$

where $\mathcal{M}_{\mathrm{BH}, 1}$ and $\mathcal{M}_{\mathrm{BH}, 2}$ are drawn from the same parent distribution for major galaxy mergers. Then,

$$
\log \left(\mu_{\text {merge }}\right)=\log \left\langle\mathcal{M}_{\mathrm{BH}, 1}+\mathcal{M}_{\mathrm{BH}, 2}\right\rangle,
$$


From propagation of errors the log-normal error is: $\sigma(\log (x))=\sigma(x) / x$, then,

$$
\sigma\left(\log \left(\mu_{\text {merge }}\right)\right)=\frac{\sigma\left\langle\mathcal{M}_{\mathrm{BH}, 1}+\mathcal{M}_{\mathrm{BH}, 2}\right\rangle}{\left\langle\mathcal{M}_{\mathrm{BH}, 1}+\mathcal{M}_{\mathrm{BH}, 2}\right\rangle},
$$

By definition, a distribution obtained by averaging the mass of merging $\mathrm{BH}$ pairs is a normal distribution with a mean of the initial parent distribution, $\mu_{\text {init }}$ :

$$
\mu_{\text {init }}=\frac{\left\langle\mathcal{M}_{\mathrm{BH}, 1}+\mathcal{M}_{\mathrm{BH}, 2}\right\rangle}{2}=\frac{\mu_{\text {merge }}}{2} .
$$

Because $\mathcal{M}_{1}$ and $\mathcal{M}_{2}$ are drawn from a normalized distribution around a parent mean $\mu$, the new distribution of $\sigma\left(\mu_{\text {merge }}\right) \equiv \sigma\left\langle\mathcal{M}_{, 1}+\mathcal{M}_{, 2}\right\rangle$ is:

$$
\sigma\left(\mu_{\text {merge }}\right) \sim \frac{\sigma\left(\mu_{\text {init }}\right)}{\sqrt{2}} \times 2 .
$$

Substituting Eqs. A4 and A5 into A3 yields:

$$
\sigma\left(\log \left(\mu_{\text {merge }}\right)\right) \sim \frac{\sigma\left(\mu_{\text {init }}\right)}{\mu_{\text {init }} \sqrt{2}} .
$$

Using the fact that, $\sigma(\log (\mu))=\sigma(\mu) / \mu$, Equation A6 becomes:

$$
\sigma\left(\log \left(\mu_{\text {merge }}\right)\right) \sim \frac{\sigma\left(\log \left(\mu_{\text {init }}\right)\right)}{\sqrt{2}} .
$$

\section{REFERENCES}

Adams, F. C., Graff, D. S., Mbonye, M., \& Richstone, D. O. 2003 ApJ, 591, 125

Adams, F. C., Graff, D. S., \& Richstone, D. O. 2001, ApJ, 551, L31

Barth, A. J., Greene, J. E., \& Ho, L. C. 2005, ApJ, 619, L151

Barth, A. J., Ho, L. C., Rutledge, R. E., \& Sargent, W. L. W. 2004, ApJ, 607, 90

Borys, C., Smail, I., Chapman, S. C., Blain, A. W., Alexander, D. M., \& Ivison, R. J. 2005, ApJ, 635, 853

Boylan-Kolchin, M., Ma, C.-P., \& Quataert, E. 2006, MNRAS, 369, 1081

Burkert, A., \& Silk, J. 2001, ApJ, 554, L151

Cattaneo, A., Blaizot, J., Devriendt, J., \& Guiderdoni, B. 2005, MNRAS, 364, 407

Cen, R. 2007, ApJ, 654, L37

Ciotti, L., Lanzoni, B., \& Volonteri, M. 2007, ApJ, 658, 65

Ciotti, L., \& van Albada, T. S. 2001, ApJ, 552, L13

Croton, D. J. 2006, MNRAS, 369, 1808

Croton, D. J., Springel, V., White, S. D. M., De Lucia, G., Frenk, C. S., Gao, L., Jenkins, A., Kauffmann, G., Navarro, J. F., \& Yoshida, N. 2006, MNRAS, 365, 11

Di Matteo, T., Colberg, J., Springel, V., Hernquist, L., \& Sijacki, D. 2007, ArXiv e-prints, 705

Di Matteo, T., Springel, V., \& Hernquist, L. 2005, Nature, 433, 604

Escala, A. 2006, ApJ, 648, L13

Ferrarese, L., Côté, P., Dalla Bontà, E., Peng, E. W., Merritt, D., Jordán, A., Blakeslee, J. P., Haşegan, M., Mei, S., Piatek, S., Tonry, J. L., \& West, M. J. 2006, ApJ, 644, L21

Ferrarese, L., \& Merritt, D. 2000, ApJ, 539, L9

Filippenko, A. V., \& Ho, L. C. 2003, ApJ, 588, L13

Fontanot, F., Monaco, P., Cristiani, S., \& Tozzi, P. 2006, MNRAS, 373,1173

Gabasch, A., Hopp, U., Feulner, G., Bender, R., Seitz, S., Saglia, R. P., Snigula, J., Drory, N., Appenzeller, I., Heidt, J., Mehlert, D., Noll, S., Böhm, A., Jäger, K., \& Ziegler, B. 2006, A\&A, 448, 101

Gebhardt, K., Bender, R., Bower, G., Dressler, A., Faber, S. M., Filippenko, A. V., Green, R., Grillmair, C., Ho, L. C., Kormendy, J., Lauer, T. R., Magorrian, J., Pinkney, J., Richstone, D., \& Tremaine, S. 2000, ApJ, 539, L13

Giallongo, E., Salimbeni, S., Menci, N., Zamorani, G., Fontana, A., Dickinson, M., Cristiani, S., \& Pozzetti, L. 2005, ApJ, 622, 116

Graham, A. W., \& Driver, S. P. 2007, ApJ, 655, 77
Graham, A. W., Erwin, P., Caon, N., \& Trujillo, I. 2001, ApJ, 563, L11

Granato, G. L., De Zotti, G., Silva, L., Bressan, A., \& Danese, L. 2004, ApJ, 600, 580

Greene, J. E., \& Ho, L. C. 2005, ApJ, 630, 122

-. 2006, ApJ, 641, L21

Haehnelt, M. G., \& Kauffmann, G. 2000, MNRAS, 318, L35

Häring, N., \& Rix, H.-W. 2004, ApJ, 604, L89

Ho, L. 1999, in ASSL Vol. 234: Observational Evidence for the Black Holes in the Universe, ed. S. K. Chakrabarti, 157-+

Ho, L. C. 2007, arXiv:0707.3436

Hopkins, P. F., Hernquist, L., Cox, T. J., Di Matteo, T., Robertson, B., \& Springel, V. 2006a, ApJS, 163, 1

Hopkins, P. F., Hernquist, L., Cox, T. J., Robertson, B., \& Krause, E. 2007, ArXiv Astrophysics e-prints

Hopkins, P. F., Somerville, R. S., Hernquist, L., Cox, T. J., Robertson, B., \& Li, Y. 2006b, ApJ, 652, 864

Islam, R. R., Taylor, J. E., \& Silk, J. 2003, MNRAS, 340, 647

-. 2004, MNRAS, 354, 427

Kaspi, S., Maoz, D., Netzer, H., Peterson, B. M., Vestergaard, M., \& Jannuzi, B. T. 2005, ApJ, 629, 61

Kaspi, S., Smith, P. S., Netzer, H., Maoz, D., Jannuzi, B. T., \& Giveon, U. 2000, ApJ, 533, 631

Kauffmann, G., \& Haehnelt, M. 2000, MNRAS, 311, 576

Kormendy, J., \& Gebhardt, K. 2001, in AIP Conf. Proc. 586: 20th Texas Symposium on relativistic astrophysics, ed. J. C. Wheeler \& H. Martel, 363-+

Kormendy, J., \& Richstone, D. 1995, ARA\&A, 33, 581

Lauer, T. R., Faber, S. M., Richstone, D., Gebhardt, K., Tremaine, S., Postman, M., Dressler, A., Aller, M. C., Filippenko, A. V., Green, R., Ho, L. C., Kormendy, J., Magorrian, J., \& Pinkney, J. 2006, ApJ, 662, 808

Lauer, T. R., Tremaine, S., Richstone, D., \& Faber, S. M. 2007, arXiv:0705.4103

Li, Y., Hernquist, L., Robertson, B., Cox, T. J., Hopkins, P. F., Springel, V., Gao, L., Di Matteo, T., Zentner, A. R., Jenkins, A., \& Yoshida, N. 2006, ArXiv Astrophysics e-prints

Magorrian, J., Tremaine, S., Richstone, D., Bender, R., Bower, G., Dressler, A., Faber, S. M., Gebhardt, K., Green, R., Grillmair, C., Kormendy, J., \& Lauer, T. 1998, AJ, 115, 2285

Marconi, A., \& Hunt, L. K. 2003, ApJ, 589, L21

McLure, R. J., \& Dunlop, J. S. 2001, MNRAS, 327, 199

Merritt, D., Milosavljević, M., Favata, M., Hughes, S. A., \& Holz, D. E. 2004, ApJ, 607, L9

Miralda-Escudé, J., \& Kollmeier, J. A. 2005, ApJ, 619, 30 
Nipoti, C., Londrillo, P., \& Ciotti, L. 2003, MNRAS, 342, 501

Onken, C. A., Ferrarese, L., Merritt, D., Peterson, B. M., Pogge, R. W., Vestergaard, M., \& Wandel, A. 2004, ApJ, 615, 645

Peng, C. Y., Impey, C. D., Ho, L. C., Barton, E. J., \& Rix, H.-W. 2006a, ApJ, 640, 114

Peng, C. Y., Impey, C. D., Rix, H.-W., Kochanek, C. S., Keeton, C. R., Falco, E. E., Lehár, J., \& McLeod, B. A. 2006b, ApJ, 649, 616

Peterson, B. M., \& Bentz, M. C. 2006, New Astronomy Review, 50, 796

Robertson, B., Cox, T. J., Hernquist, L., Franx, M., Hopkins, P. F., Martini, P., \& Springel, V. 2006a, ApJ, 641, 21

Robertson, B., Hernquist, L., Cox, T. J., Di Matteo, T., Hopkins, P. F., Martini, P., \& Springel, V. 2006b, ApJ, 641, 90

Salviander, S., Shields, G. A., Gebhardt, K., \& Bonning, E. W. 2006, New Astronomy Review, 50, 803

—. 2007, ApJ, 662, 131

Schechter, P. 1976, ApJ, 203, 297

Shields, G. A., Gebhardt, K., Salviander, S., Wills, B. J., Xie, B. Brotherton, M. S., Yuan, J., \& Dietrich, M. 2003, ApJ, 583, 124
Shields, G. A., Salviander, S., \& Bonning, E. W. 2006, New Astronomy Review, 50, 809

Sijacki, D., Springel, V., Di Matteo, T., \& Hernquist, L. 2007, ArXiv e-prints, 705

Silk, J., \& Rees, M. J. 1998, A\&A, 331, L1

Soltan, A. 1982, MNRAS, 200, 115

Springel, V., Di Matteo, T., \& Hernquist, L. 2005, MNRAS, 361, 776

Treu, T., Malkan, M. A., \& Blandford, R. D. 2004, ApJ, 615, L97

Treu, T., Woo, J.-H., Malkan, M. A., \& Blandford, R. D. 2007, ArXiv e-prints, 706

Vestergaard, M. 2002, ApJ, 571, 733

Vestergaard, M., \& Peterson, B. M. 2006, ApJ, 641, 689

Volonteri, M., Haardt, F., \& Madau, P. 2003, ApJ, 582, 559

Volonteri, M., \& Perna, R. 2005, MNRAS, 358, 913

Woo, J.-H., Treu, T., Malkan, M. A., \& Blandford, R. D. 2006, ApJ, 645, 900

Yu, Q., \& Tremaine, S. 2002, MNRAS, 335, 965 